\title{
FAST NUMERICAL MODELLING METHOD FOR WIND FLOW INVESTIGATION BASED ON DEPTH-AVERAGED EQUATIONS
}

\author{
ANNA AVRAMENKO ${ }^{1}$, OXANA AGAFONOVA ${ }^{1}$, JOONAS SORVARI ${ }^{1}$, \\ HEIKKI HAARIO $^{1} \&$ YURY AVRAMENKO ${ }^{2}$ \\ ${ }^{1}$ Lappeenranta University of Technology, School of Engineering Science, Finland \\ ${ }^{2}$ Moscow State Teachers' University, Institute of Mathematics and Informatics, Russian Federation
}

\begin{abstract}
An important phase of wind farm design is solving the wind farm layout optimization problem, which consists in optimally positioning the turbines within the wind farm so that the wake effects are minimized and therefore the expected power production maximized. Simulating a wind farm with more than one fully detailed wind turbine and possibly complex terrain geometry requires significant computational power and time. This paper concerns the study of the depth-averaged flow of the wind turbine with a CFD-RANS approach. The complex three-dimensional (3D) geometry need not be modelled or discretized in the pre-processing state: instead, the geometry of the terrain is only described with source terms in the depth-averaged equations, which are then solved in a very simple and fixed two-dimensional (2D) domain. This approach reduces the equations from 3D to 2D and decreases the elapsed time of CFD simulations from hours to minutes. Thus, it is a very practicable modelling method in real time optimization work. 2D CFD simulations are compared with full 3D models and experiments. This paper gives an overview of the existing work and discusses the challenges that may be overcome by future research.
\end{abstract}

Keywords: wind farm, depth-averaged equations, CFD, optimization.

\section{INTRODUCTION}

Wind energy is widely becoming recognized as one of the most cost-efficient sources of renewable energy. Therefore, expectations for wind energy are at important levels as the goal is for wind energy to become a prevailing source for global electricity needs. One of the keys to realizing this goal in a cost-efficient manner is to utilize existing wind farms in a more efficient manner through improved control algorithms [1].

Wind farm layout optimization is a complex problem and recent growth of large wind farms has increased demands on designers. Typically, the problem has been cast as a geometric optimization problem, usually on a discrete grid, using a power-based cost function. Direct search approaches have been employed wherein specific constraints of the problem are translated into a predefined fitness function and conditions, e.g. wind speed data, geometry of the terrain and turbine dimensions, are inputs. Once this configuration of the algorithm is set up by the wind farm designer, 3D CFD simulations takes some days to converge, and must be run again if the problem changes [2].

This paper presents a novel depth-averaged approach that facilitates wind farm optimization where simulations are available in a matter of minutes regardless of different constraints and conditions.

Depth-averaged flow equations are mostly used to simulate shallow water flows. In this kind of flows the vertical characteristic length is much smaller that the horizontal one. Shallow water equations have been used for high Reynolds number turbulent water flows and mostly for open channels like in the modelling of a tidal flow in complex estuaries [3] and for water flows in a lake or in a sea [4]. Depth-averaged equations have been validated for flows in a closed channel, such as in a head box of a paper machine [5], [6]. In 
addition, this method was firstly used for optimization of Chevron-type plate heat exchangers by Lyytikainen [7] and it presents the general flow behaviour with good accuracy.

Fast modelling for optimization is accomplished by depth-averaged flow that is derived by integrating the original 3D Navier-Stokes equations over the height of the domain. This approach reduces the equations from $3 \mathrm{D}$ to $2 \mathrm{D}$. The goal of the derived modelling method is to reduce the computational time of CFD simulations from hours to minutes. With this approach, only one 2D mesh should be generated and the depth of the domain can be presented in every horizontal position with the source terms in governing equations. Thus, geometry pre-processing work can be skipped.

Optimization of the wind turbines will be accomplished with evolutionary algorithms to avoid difficulties in gradient calculations. Overall, evolutionary algorithms are shown to be very convenient for our optimization case even though they require a lot of CFD simulations during the optimization process [7]. Power maximization of wind turbines in the wind farm was selected to give information for wind farm enhancement requirements:

$$
P=\frac{\rho U^{3}}{2} \pi R^{2} C_{p}
$$

where $\mathrm{P}$ is a power of the turbine, $\rho$ is the density, $\mathrm{U}$ is the velocity, $\mathrm{R}$ is the radius of turbine and $C_{p}$ is a power coefficient. Total power of wind farm will be used in future as the objective function for evolutionary algorithms. Therefore, only velocity components should be clear estimated in $2 \mathrm{D}$ and $3 \mathrm{D}$ wind farm simulations, other variables as pressure, turbulent quantities and etc. are not so necessary for this work.

A full 3D and 2D CFD-RANS approach was used, modelling a wind turbine by means of Actuator Disk Model using Porous Media Model in ANSYS Fluent. Both the near and far wake was analyzed. A detailed selection of results is presented, dealing with the various investigated issues. The depth-averaged model, which is fast but not so accurate, will be used in future in the optimization procedure and the accurate 3D CFD model is used to validate our fast model. 3D modelling is validated with experiments also.

\section{METHODOLOGY}

\subsection{Depth-averaged equations}

Simulations in this work have been performed with ANSYS Fluent. Therefore, depthaveraged conservation laws were derived in the same form as standard 2D-equations in ANSYS Fluent, such that all extra terms are included in the source terms. The depthaveraging were performed along the vertical or $\mathrm{z}$ direction between the bottom level $d_{1}(x, y)$ and the top level $d_{2}(x, y)$. The depth-averaged velocity components $U(x, y)$ and $V(x, y)$ are thus defined as:

$$
U=\frac{1}{D} \int_{d_{1}}^{d_{2}} u d z, \quad V=\frac{1}{D} \int_{d_{1}}^{d_{2}} v d z
$$

where $D(x, y)=d_{2}(x, y)-d_{1}(x, y)$ is the height of the domain, $\vec{u}=(u, v, w)$ is the velocity vector in $3 \mathrm{D}$ domain. The geometry is presented by the long parallelepiped, where the bottom surface is a rough terrain and the top surface is straight one, therefore $d_{2}(x, y)$ is constant. The symmetry boundary condition was applied on the top and the wall boundary condition was used for the terrain. The pressure was approximated as a constant in the zdirection. 
The whole derivation of depth-averaged equations can be found in [8], only the final mass and momentum conservation laws are presented in this paper:

$$
\begin{gathered}
\nabla \cdot(\rho \vec{U})=-\frac{\rho}{D} \vec{U} \cdot \nabla D=S_{m}, \\
\nabla \cdot(\rho \vec{U} \vec{U})=-\nabla \mathrm{p}+\nabla \cdot(\overline{\bar{\tau}})+\rho \vec{g}+\vec{U} S_{m}-\frac{32 \mu \vec{U}}{D^{2}},
\end{gathered}
$$

where $\mu$ is the dynamic viscosity, $p$ is the pressure, $\rho$ is the density, $\vec{U}$ is the depth-averaged velocity vector, $\overline{\bar{\tau}}$ is the stress tensor. In addition, it has to be noted, that velocities are depth-averaged velocities so that the depth-averaged velocity vector is $\vec{U}=(U, V)$.

Derived mass balance and momentum equations are sufficient for laminar flow. Otherwise, turbulence quantities should be simulated. The simplified production terms for turbulence and its dissipation can be obtained from [9]. $\kappa$ and $\varepsilon$ equations cannot be integrated over the height so that they assume a closed form, where source terms can be written as:

$$
S_{k}=\rho c_{k} \frac{u_{f}^{3}}{D}, S_{e}=\rho c_{e} \frac{u_{f}^{4}}{D^{2}},
$$

where $u_{f}$ is the frictional velocity, $c_{e}, c_{k}$ are empirical parameters. Now the source terms for mass balance, momentum equations and the turbulence model have been introduced. These sources are used in simulating a wind flow in the wind farm with depth-averaged governing equations.

\subsection{Actuator Disc Model}

Conducting detailed analyses of a single Horizontal Axis Turbine (HAT) is problematic as this requires detailed turbine design and performance information which is likely to be commercially restricted. An equivalent actuator disk model representing a real turbine in a three-dimensional CFD model offers an economically viable analysis tool, in terms of mesh size and solution timescales, for assessing turbine response in a range of conditions and environments [10]. The properties of the wake behind a three-blade rotating wind turbine and behind a porous disc generating a similar velocity deficit were compared through wind tunnel experiments in [11]. Results have shown that the mean velocity deficit, the stream wise turbulence intensity, the stream-wise skewness and Kurtosis, at the three-diameter of the turbine downstream of a wind turbine and of a porous disc are closely similar in high intensity turbulent inflow conditions. Therefore, the turbine in this work was modelled in the computational domain as an actuator disk.

The Porous Media Model in ANSYS Fluent is used to implement ADM. Porous Media are modelled by the addition of a momentum source term to the standard fluid flow equations. The source term is composed of two parts: a viscous loss term and an inertial loss term, and for simple homogeneous porous media can be presented in the next form:

$$
S_{i}=-\frac{\mu}{\alpha} u_{i}-C_{2} \frac{1}{2} \rho u_{i}|\vec{u}|,
$$

where $S_{i}$ is the source term for $i$-th (x, y, z) momentum equation, $\vec{u}$ is the velocity vector. According to the theory of ADM, the viscous loss term is equal to zero [12]. Thus, only the inertial resistance factor is needed to implement Porous Media Model.

The present study focuses on the numerical investigation of the wake development in a Nordtank NTK 500/41 wind turbine with LM 19.1 m blades described in Mikkelsen's thesis [13]. Relevant data for the blade and the case are the total radius of the blade is 
$20.5 \mathrm{~m}$, the power coefficient $c_{p} \approx 0.4$, the free-stream velocity $u_{0}=10 \mathrm{~m} / \mathrm{s}$. The inertial resistance factor for this case can be obtained from the next algorithm:

1. The axial induction factor, $a$, can be found from a given power coefficient of the wind turbine, $c_{p}: c_{p}=4 a\left(1-a^{2}\right)$.

2. Then, the far downstream velocity, $u_{w}$, and the velocity at the disk, $u_{D}$, are calculated using the free-stream velocity: $u_{w}=u_{0}(1-2 a), u_{D}=u_{0}(1-a)$.

3. The pressure jump can be obtained using the next equation: $\Delta p=\frac{1}{2} \rho\left(u_{0}^{2}-u_{w}^{2}\right)$.

4. From the other hand, $\Delta p=-S \Delta m=\frac{1}{2} C_{2} \rho u_{D}^{2} \Delta m$, where $\Delta m$ is thickness of the porous disk.

The thickness of the turbine can be taken as $1 \mathrm{~m}$ [14], therefore $C_{2}=0.6136$ according to the previous algorithm.

The main problem for fast numerical modelling tool based on depth-averaged equations is to simulate the wind turbine in $2 \mathrm{D}$ case. Unfortunately, it is not possible to make simulation of wind turbine in general way for 2D modelling. Therefore, a new approach was developed especially for this case using actuator disc model. The main idea of this method is that the depth-averaged velocity obtained from 3D simulation should coincide with the results of $2 \mathrm{D}$ modelling. The turbine is presented as a rectangle where its length is the thickness of the turbine and the width - the diameter. It should be noted, it is not possible to build mesh for the wind turbine each time when position of the turbine is changed during wind farm optimization. The mesh building is time-consuming for the optimization. Therefore, Porous Zone Model, described as eqn. (6), is applied to this region using User-Defined Functions. The viscous loss term is also 0, but the inertial resistance factor for this model differs from the same coefficient for 3D model, obtained above. $C_{2}$ is found numerically by comparing 3D and 2D simulations and it equals 0.11 .

The detailed description of the simulation of the wind turbine on the smooth terrain is given in [8]. The depth-averaged velocity in 3D simulation is compared with the velocity in 2D numerical modelling in this work. The results presented in this paper show good accuracy of depth-averaged velocity in $2 \mathrm{D}$ and $3 \mathrm{D}$ cases. It means, that this method can be combined with depth-averaged equations to simulate the wind turbine on the complex terrain.

\section{RESULTS}

The modelling method, developed in this work, was used to compare average velocity for various terrain designs. The overall accuracy of the model is essential, but detailed pointwise velocities, pressures or turbulence quantities are not in main focus. $\kappa-\varepsilon$ RANS simulation were performed using ANSYS Fluent. RANS models solve averaged NavierStokes equations and thus provide average solutions. In other words, they do not compute turbulent fluctuations at all but consider turbulence effects on mean flow values.

Two different cases were considered: simulation of the flow field over the one hill and simulation of the wind turbine on a hill. 3D and 2D results of numerical modelling were compared for all cases, but for the first case, they were confirmed also by the experiment, described below.

\subsection{Simulation of the flow field over the one hill}

Laboratory Experiment by Khurshudyan, described in [15], was used to compare numerical results. In this experiment, flow measurements were made around two-dimensional symmetric hills of varying steepness. The majority of measurements were made with the 
same hill height, $\mathrm{H}=0.117 \mathrm{~m}$, and with a free-stream wind speed $U_{\infty}=4 \mathrm{~m} / \mathrm{s}$, giving a Reynolds number $R e=3.1 \times 10^{4}$. A normalized friction velocity was equal to $u_{*} / U_{\infty}=$ 0.0445 . At the hill location, a depth of the boundary layer was about $1 \mathrm{~m}$ with a roughness length $z_{0}=0.157 \mathrm{~mm}$. The hills were designated H3, H5 and H8 according to their length/height ratio $\mathrm{a} / \mathrm{H}$ and their shape was given parametrically by:

$$
\begin{gathered}
x=\frac{1}{2} \xi\left[1+\frac{a^{2}}{\xi^{2}+m^{2}\left(a^{2}-\xi^{2}\right)}\right], \\
y=\frac{1}{2} m \sqrt{a^{2}+\xi}\left[1-\frac{a^{2}}{\xi^{2}+m^{2}\left(a^{2}-\xi^{2}\right)}\right],|\xi| \leq a,
\end{gathered}
$$

where $m=n+\sqrt{n^{2}+1}$ and $n=H / a$ is average sloop.

The hill H3 is considered in this paper, because flow separation was observed on the lee slope of hill H3 in the wind-tunnel study. This was confirmed by the computations undertaken here.

RANS simulation was performed in $10 \mathrm{~m} \times 1 \mathrm{~m}$ horizontal and $1 \mathrm{~m}$ vertical domain, in which $\mathrm{x}$ is stream wise direction and $\mathrm{z}$ the vertical one. The computational domain was created using a hexahedral mesh. In the near wall region, the 3D mesh has been refined in the stream wise direction in the area around the hill crest so as to better resolve the flow around the separation point. Additionally, this grid was slightly stretched normal to the lower wall. Here, the notation of ANSYS Fluent [16] is used for the definition of the boundary conditions:

- Inlet: Velocity-inlet Logarithmic profile.

$$
\begin{gathered}
U=\frac{u_{*}}{K} \ln \frac{z}{z_{0}} \quad \text { if } z \leq H, \\
U=U_{\infty}, \text { if } z>H,
\end{gathered}
$$

where $K=0.41$ is Karman's constant. Turbulent kinetic energy $\kappa=0.0012$ and turbulent dissipation rate $\varepsilon=9.591 e-06$ are obtained from experiment.

- Outlet: Outflow.

- Lower bound: Wall.

- Upper bound: Symmetric.

- Lateral bounds: Periodic.

The computational domain for 2D simulation was $10 \mathrm{~m}$ long (X-axis) by $1 \mathrm{~m}$ wide (Yaxis). The mesh consisted approximately 150,000 quad elements. At the inlet face, undisturbed uniform wind velocity was fixed at $3.81 \mathrm{~m} / \mathrm{s}$, outflow was set at the outlet and periodic condition was selected for the lateral walls.

Figs 1 and 2 present results of stream wise and depth-averaged velocity profiles for 3D and 2D models accordingly. Fig. 1 shows also a flow separation on the lee side of the hill that was expected from the experiment.

Fig. 3 shows mean-velocity profiles (x-component) computed in $3 \mathrm{D}$ simulation at the upwind base, summit and downwind base. In each case, the agreement with the experiment is good, particularly in respect to the maximum speed-up at the summit. This confirms the effectiveness of comparatively simple turbulence models in predicting the dominant shear stress - and hence the mean flow - in perturbed deep boundary layers.

The depth-averaged velocity values of 3D simulations calculated by eqn (2) for five different points of the hill: before, after and in the middle of the hill. Table 1 presents results of depth-averaged velocities obtained from the experiment and numerical modelling for these points. It can be seen, that average velocity values are accurately predicted in using the depth-averaged model. 

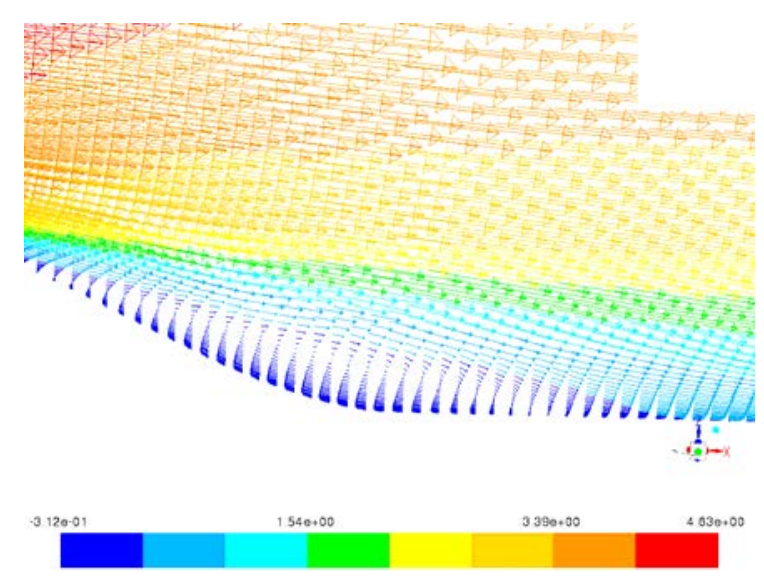

Figure 1: Contours of stream wise velocity for 3D flow modelling over the hill in a vertical plane perpendicular to the hill.
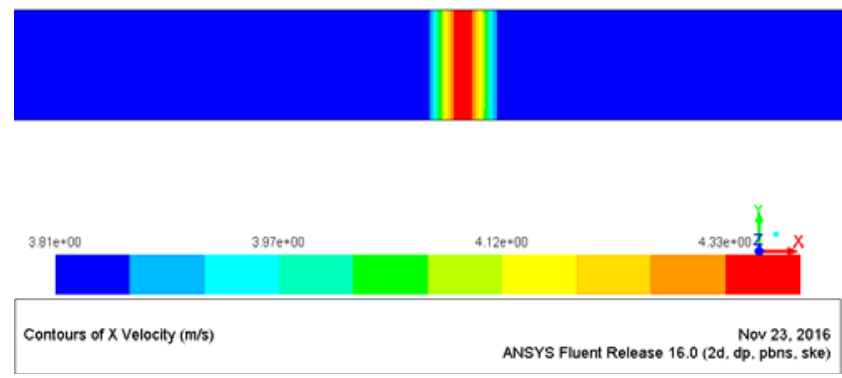

Figure 2: Contours of depth-averaged velocity for 2D flow simulation over the hill.
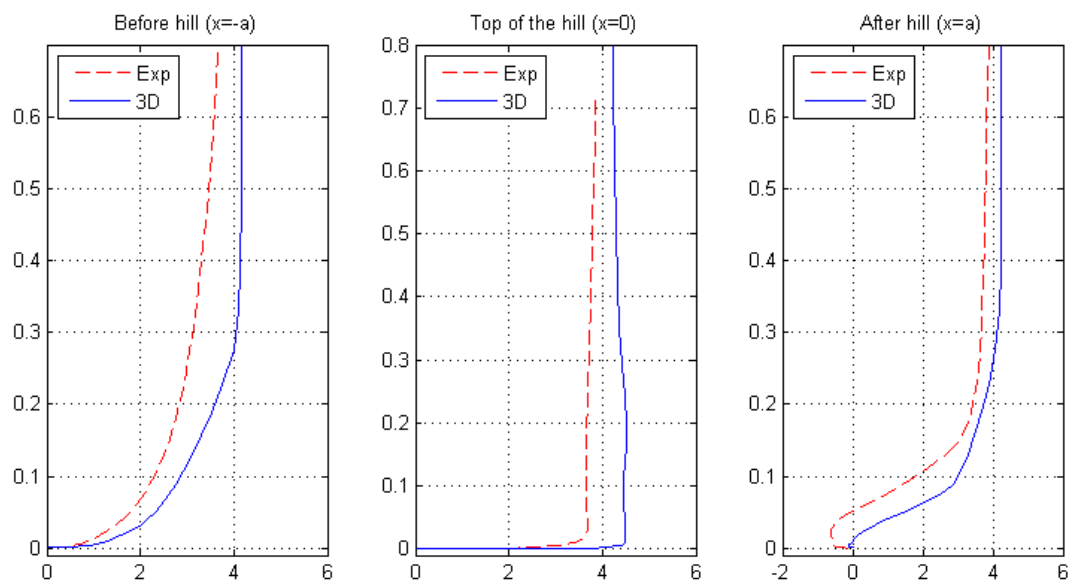

Figure 3: Experiment: comparison of velocity profiles over hill H3. 
Table 1: Depth-averaged velocity for flow simulations over the hill.

\begin{tabular}{|l|l|l|l|}
\hline \multirow{2}{*}{$\mathrm{X}$} & \multicolumn{4}{|l|}{ Depth-averaged velocity obtained from: } \\
\cline { 2 - 4 } & Experiment & $3 \mathrm{D}$ & $2 \mathrm{D}$ \\
\hline-0.351 & 3.217 & 3.836 & 3.827 \\
\hline-0.175 & 3.755 & 4.125 & 4.116 \\
\hline 0 & 3.923 & 4.340 & 4.330 \\
\hline 0.176 & 3.657 & 4.124 & 4.114 \\
\hline 0.351 & 3.337 & 3.837 & 3.827 \\
\hline
\end{tabular}

3.2 Flow simulation of the wind turbine on the hill

In this part, simulations used to compute the wind fields of the Nordtank NTK 500/41 wind turbine on the hill, described by:

$$
z=H e^{-\frac{(x-500)^{2}+(y-400)^{2}}{3000}},
$$

where $H=60 \mathrm{~m}$ is the height of the hill.

The 3D simulations were performed using a domain of $550 \times 260 \times 200$ elements with dimensions of $2000 \mathrm{~m} \times 800 \mathrm{~m} \times 400 \mathrm{~m}$ (wind direction, cross-wind direction and vertical direction). A hexahedral mesh of approximately 20 million volumes was created. Around the actuator disc, the grid was H-shaped and it was optimized to achieve sufficient resolution. Also, the mesh has been refined along the hill to model the boundary layer with the help of wall functions.

The boundary conditions were: uniform stream wise velocity at the inlet and outflow boundary condition at the outlet. The lateral walls of the domain were modelled as periodical, the symmetry condition was set at the top wall. The turbine was modelled in the computational domain as an actuator disk using Porous Zone Mode, described by eqn (6).

The computational domain for 2D simulation was $2000 \mathrm{~m}$ long (X-axis) by $800 \mathrm{~m}$ wide (Y-axis). The mesh consisted approximately 600000 quad elements. The boundary condition was the same as for 3D simulation: velocity $10 \mathrm{~m} / \mathrm{s}$ at the inlet, outflow at the outlet, other walls are periodical.

Fig. 4 shows the velocity-vector field across the centre-plane of the hill in 3D modelling and Fig. 5 is presented velocity distribution in $2 \mathrm{D}$ wind turbine simulation on the hill.

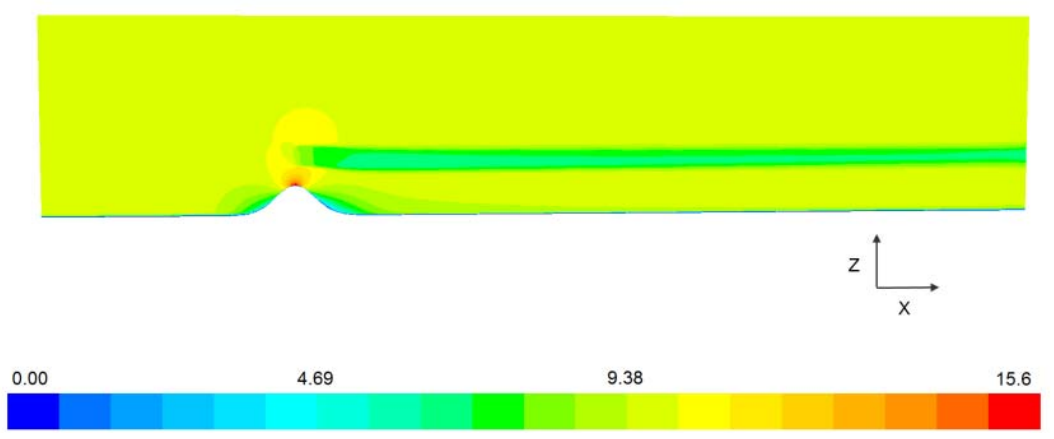

Figure 4: Flow on centre-plane of the hill for 3D simulation. 


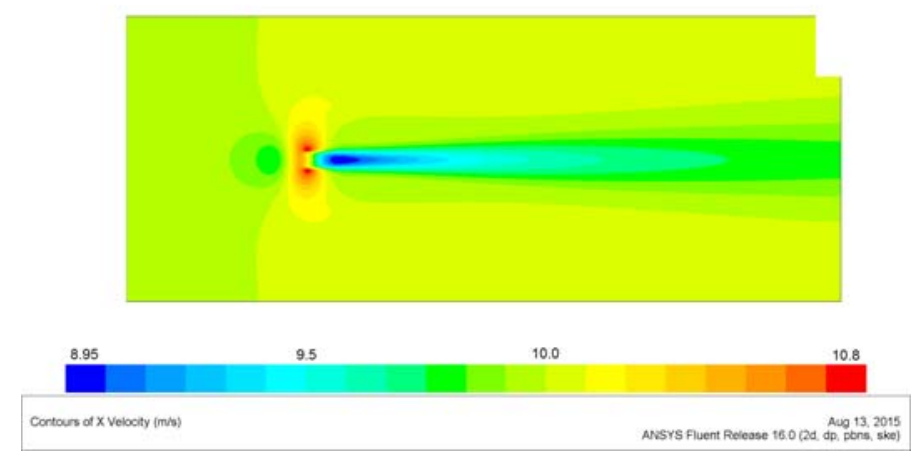

Figure 5: Depth-averaged velocity distribution in 2D wind turbine simulation on the hill.

Table 2: Depth-averaged velocity obtained for wind turbine simulations on the hill.

\begin{tabular}{|l|l|l|l|}
\hline$X$ & Y & \multicolumn{2}{|l|}{ Depth-averaged velocity obtained from: } \\
\cline { 3 - 4 } & & 3D & 2D \\
\hline 200 & 400 & 9.9421 & 9.98324 \\
\hline 500 & 400 & 10.2889 & 10.3415 \\
\hline 700 & 400 & 9.15 & 9.12865 \\
\hline 1500 & 400 & 9.6181 & 9.72692 \\
\hline 500 & 200 & 10.0624 & 10.107 \\
\hline 500 & 380 & 10.4728 & 10.4108 \\
\hline
\end{tabular}

The depth-averaged velocity values of $3 \mathrm{D}$ simulations calculated by eqn. (2) for six different points of the hill: the middle of the hill, after the hill in the wind direction and after the hill in the cross-wind direction. Table 2 presents results of comparison between 3D and 2D depth-averaged velocity components for wind turbine simulations on the hill. In the light of validations discussed in these sections, the depth-averaged model is sufficiently accurate for the velocity calculations, but one should be cautious about using the depthaveraged model for detailed predictions of point-wise velocity components, pressure and other variables.

\section{CONCLUSION}

The investigation of the wind flow around turbine is the key issue in the product development of wind farms. CFD is a very promising tool to study wind behaviour, but still one major challenge is the complex geometry of the wind farm. Modelling the detailed geometry and generating a high-quality mesh, is extremely time consuming. Therefore, the development of faster modelling methods is of utmost importance in order to produce tools for real optimization work. Replacing the full 3D flow by the depth-averaged equations makes it possible to save both human and CPU time. Using the depth-averaged approach the complex 3D geometry need not be modelled or discretized: instead, the geometry of the terrain is only described with source terms in the depth-averaged equations, which are then solved in a very simple and fixed $2 \mathrm{D}$ domain. The method is also very fast from the point of view of a computational time. Even though the depth-averaged equations lose some 3D flow characteristics, they are capable of presenting the general flow behaviour with surprisingly good accuracy. Further, they speed up the optimization process and help in 
finding the best position of the wind turbines in the wind farm, which can be then examined with 3D modelling in more detail. The method introduced in this paper gives a good basis for the further development of the fast and efficient modelling of the wind farm.

\section{REFERENCES}

[1] Marden, J.R., Ruben, S.D. \& Pao, L.Y., Surveying Game Theoretic Approaches for Wind Farm Optimization. Proceedings of the AIAA Aerospace Sciences Meeting, 2012.

[2] Wilson, D., Cussat-Blanc, S., Veeramachaneni, K., O’Reilly, U.M. \& Luga, H., A Continuous Developmental Model for Wind Farm Layout Optimization. Proceedings of the Genetic and Evolutionary Computation Conference, 2014.

[3] Cea, L., French, J. \& Vazquez-Cendon, M., Numerical modelling of tidal flows in complex estuaries including turbulence: An unstructured finite volume solver and experimental validation. International Journal for Numerical Methods in Engineering, 67, pp. 1909-1932, 2006.

[4] Solbakov, V., Application of Mathematical Modelling for Water Environment Problems. PhD thesis, University of Jyvaskyla, Finland, 2004.

[5] Thévenin, D. \& Janiga, G., Optimization and Computational Fluid Dynamics, pp. 110-127, 2008.

[6] Hamalainen, J. \& Tiihonen, T., Modelling and simulation of fluid flows in a paper machine headbox. Proceedings of the ICIAM 95, Issue 4: Applied sciences, especially mechanics (minisymposia), pp. 62-66, 1995.

[7] Lyytikainen, M., Modelling and Optimising of Chevron-type Plate Heat Exchangers. $\mathrm{PhD}$ thesis, University of Kuopio, Finland, 2009.

[8] Avramenko, A., Agafonova, O. \& Sorvari, J., Heikki, H., Numerical modelling of flow around a wind turbine using a fast method based on depth-averaged equations. Global Journal of Pure and Applied Mathematics, 11(6), pp. 5057-5073, 2015.

[9] Rodi, W., Turbulence Models and Their Application in Hydraulics, A State of the Art Review, Ashgate Pub Co., 1984.

[10] Bai, L., Spence, R.R.G. \& Dudziak, G., Investigation of the influence of array arrangement and spacing on tidal energy converter (TEC) performance using a 3dimensional CFD model. Proceedings of the 8th European wave and tidal energy conference, 2009.

[11] Aubrun, S., Loyer, S., Hancock, P.E. \& Hayden, P., Wind turbine wake properties: Comparison between a non-rotating simplified wind turbine model and a rotating model. Journal of Wind Engineering and Industrial Aerodynamics, 120, pp. 1-8, 2013.

[12] Adamski, S.J., Numerical Modelling of the Effects of a Free Surface on the Operating Characteristics of Marine Hydrokinetic Turbines. Master of Science in Mechanical Engineering, University of Washington, 2013.

[13] Mikkelsen, R., Actuator Disc Methods Applied to Wind Turbines. PhD thesis, Technical University of Denmark, 2003.

[14] Svenning, E., Implementation of an actuator disk in OpenFOAM. Bachelor thesis, Chalmers University of Technology, Sweden, 2010.

[15] Khurshudyan, L.H., Snyder, W.H. \& Nekrasov, I.V., Flow and dispersion of pollutants over two-dimensional hills. United States Environmental Protection Agency Report EPA-600/4-81- 067, 1981.

[16] User's Guide: ANSYS Fluent 12.0 User's Guide, pp. 7.56-7.168, NH, USA, 2009. 